\title{
Dislocated quasi-metric stability of a multiplicative inverse functional equation
}

\author{
B. V. Senthil Kumar ${ }^{a, *}$, Khalifa Al-Shaqsia ${ }^{a}$, S. Sabarinathan ${ }^{b}$ \\ ${ }^{a}$ Department of Information Technology, University of Technology and Applied Sciences, Nizwa - 611, Oman. \\ ${ }^{b}$ Department of Mathematics, Faculty of Engineering and Technology, SRM Institute of Science and Technology, Kattankulathur - 603 \\ 203, Tamil Nadu, India.
}

\begin{abstract}
In this study, we employ the dislocated metric space stability result of an equation with one variable function to prove different stabilities of a two variable equation involving rational function in the codomain of complete dislocated quasi-metric spaces. We also extend the stabilities by taking different upper bounds.
\end{abstract}

Keywords: Functional equation, multiplicative inverse functional equation, Ulam stability, dislocated quasi-metric space. 2020 MSC: 39B82, 39B72.

(C)2022 All rights reserved.

\section{Introduction and preliminaries}

The role of dislocated metric spaces is very significant in cybercrime and cryptography for protecting data and information. In particular, the applications of these spaces are of great importance in elliptic curve cryptography [35]. Hence, this raises many more applications in information and communication technologies and other domains of computing. Let us revocate few essential ideas of dislocated metric spaces.

Let $B$ be a non-empty set with a metric $d: B \times B \longrightarrow \mathbb{R}^{+} \cup\{0\}$, which satisfies the subsequent conditions:

(i) $d(v, \xi)=d(\xi, v)=0 \Longrightarrow v=\xi$;

(ii) $d(\nu, \xi) \leqslant d(\nu, \tau)+d(\tau, \xi)$

for all $v, \xi, \tau \in B$. The mapping $d$ defined with the set $B$ is called a dislocated quasi-metric (in short dqmetric) and the pair $(B, d)$ is said to be a dislocated quasi-metric space (in short dq-metric space) (Refer $[37,49])$. The pair $(B, d)$ is called a metric-like space if the dq-metric $d$ defined in $B$ satisfies the symmetric condition, that is, $d(v, \xi)=d(\xi, v)$ for $v, \xi \in B$ (see [6]).

\footnotetext{
*Corresponding author

Email addresses: senthilkumar@nct.edu.om (B. V. Senthil Kumar), khalifa.alshaqsi@nct.edu.om (Khalifa Al-Shaqsi), ssabarimaths@gmail . com (S. Sabarinathan)
}

doi: $10.22436 /$ jmcs.024.02.05

Received: 2020-12-26 Revised: 2020-01-01 Accepted: 2020-01-05 
Example 1.1. A mapping $d: \mathbb{R}^{2} \longrightarrow \mathbb{R}^{+} \cup\{0\}$ defined via $d(\nu, \xi)=|v|$ for $v, \xi \in \mathbb{R}$ is a dislocated quasi-metric.

Example 1.2. A mapping $d: \mathbb{R}^{2} \longrightarrow \mathbb{R}^{+} \cup\{0\}=\max \{|v|,|\xi|\}$ for $\nu, \xi \in \mathbb{R}$, then the pair $(\mathbb{R}, d)$ is a metriclike space.

Suppose $(B, d)$ is a dq-metric space and suppose $\left\{v_{m}\right\}$ is a sequence of elements in $B$. Then the limit of the sequence $\left\{v_{m}\right\}$ converges to $v \in B$ if and only if $d\left(v_{m}, v\right) \rightarrow 0$ and $d\left(v, v_{m}\right) \rightarrow 0$ and it can be verified that this limit if it exists then it is unique. If the sequence $\left\{v_{m}\right\}$ converges to $v$, then we can write it as $v_{m} \rightarrow v$. Precisely, suppose the there are two different limits $\alpha, \beta \in B$ of the sequence $\left\{v_{m}\right\}$, then we have $0 \leqslant d(v, \xi) \leqslant d\left(v, \xi_{m}\right)+d\left(v_{m}, \xi\right)$. This implies that $d(v, \xi)=0$ and analogously, we have $d(\xi, v)=0$, and hence we get $v=\xi$.

Let $\left\{v_{m}\right\}$ be a sequence. Then it is said to be Cauchy if for all $\epsilon>0$, there exists $M \in \mathbb{N}$, for all $r, s \geqslant M, d\left(v_{s}, v_{r}\right)<\epsilon$. If every Cauchy sequence is convergent in a dq-metric space $(B, d)$, then it is said to be complete.

The classical stabiity result of a one variable functional equation is obtained in the following theorem. The proof of this theorem is available in [17]. This theorem plays a crucial key to prove our major results. The following notations are used in the theorem. Let $(B, d)$ be a dq-metric space with completeness, $L$ be a non-empty set, $\Phi: \mathrm{B} \longrightarrow \mathrm{B}, \mathrm{g}: \mathrm{L} \longrightarrow \mathrm{L}$, and $\mathrm{f}_{\mathrm{k}}: \mathrm{L} \longrightarrow \mathbb{R}^{+} \cup\{0\}$ be mappings. Suppose the mapping $\Phi$ satisfies the Lipschitz condition $d(\Phi(r), \Phi(s)) \leqslant \mu d(r, s)$ for all $r, s \in B$ and for $k=1,2$, where $\mu>0$ is a Lipschitz constant, $F_{k}(r)=\sum_{k=0}^{\infty} \mu^{k} f_{k}\left(g^{k}(r)\right)<\infty$ for every $r \in L$.

Theorem 1.3. Suppose a mapping $\mathrm{h}: \mathrm{L} \longrightarrow \mathrm{B}$ satisfies the following inequalities

$$
d(\Phi \circ h \circ g(r), h(r)) \leqslant f_{1}(r), \quad r \in L, \quad d(h(r), \Phi \circ h \circ g(r)) \leqslant f_{2}(r), \quad r \in L .
$$

Then a mapping $\mathrm{H}: \mathrm{L} \longrightarrow \mathrm{B}$ exists defined by the existence of the limit $\mathrm{H}(\mathrm{r})=\lim _{\mathrm{k} \rightarrow \infty} \Phi^{\mathrm{k}} \circ \mathrm{h} \circ \mathrm{g}^{\mathrm{k}}(\mathrm{r})$ for all $\mathrm{r} \in \mathrm{L}$ and satisfies the equation $\Phi \circ \mathrm{H} \circ \mathrm{g}=\mathrm{H}$ such that

$$
d(H(r), h(r)) \leqslant F_{1}(r), \quad r \in L, \quad d(h(r), H(r)) \leqslant F_{2}(r), \quad r \in L .
$$

The most active research area in the domain of analysis is stability theory. The investigation of validity of stabilities of functional equations has become the crucial question for various research problems and a number of motivating and novel results are published by many mathematicians. The vital question raised in [56] became the backbone for the evolution of stability theory of equations. This fundamental question is concerned with determining approximate group homomorphisms. The question can be stated as "When an approximate homomorphism exists near to the given homomorphism?" This question triggered to find the solution and it was first responded in [20] by considering fundamental Cauchy additive equation $\chi(\nu+\xi)=\chi(\nu)+\chi(\xi)$ in the framework of Banach spaces. Later on, the results obtained in [20] were dealt in different versions by many mathematicians [7, 10, 15, 16, 38, 40, 57]. A lot of fundamental ideas about functional equations and inequalities are published as books $[5,9,13,28,39$, $41,48]$. The stability results of various other forms of functional equations are published in the form of monographs in $[21,24]$. There are lot of interesting information regarding stability results available in $[11,12,14,22,23,29,31,45-47]$. Quite recently for the past one decade, there are new and interesting stability results of several functional equations are obtained in $[3,4,25-27,32-34,36]$.

The pioneering multiplicative inverse functional equation in the dynamic research domain of stability theory of equations is

$$
h(r+s)=\frac{h(r) h(s)}{h(r)+h(s)}
$$

The solution and stability results of equation (1.1) were obtained in [43]. Later on, there are numerous intriguing and noteworthy stability results, applications and intepretations of various forms of multiplicative inverse functional equations are published in $[8,18,30,42,50,51]$. The solutions of these 
multiplicative inverse functional equations are of rational form provide exceptional contributions in various disciplines such as to compute the combined resistance of a parallel electric circuit with resistors [44], to study physical properties of objects [52], to interpret them with a significant hypothesis arising in electromagnetic theory and the relation of stiffness and length of diving board [53], to compute the total focal length of combined lenses in optics [54], to interpret through an application in electromagnetism [55].

The considerable contribution of these type functional equations in other spheres and the remarkable results obtained in Theorem 1.3 influenced us to deal with the same approach to the stablesness of equation (1.1). Hence, in this article, by the approach of Theorem 1.3, we prove the stabilities of equation (1.1) where $h: L \longrightarrow B$ is a mapping with $L$ as a non-empty set and $B$ as a dq-metric space. We also extend further stability results by taking different upper bounds.

\section{Stability of equation (1.1) via the approach of Theorem 1.3}

In this section, we apply the approach applied in Theorem 1.3 to prove stability results of equation (1.1). Let us consider $(A,+)$ is a square summetric groupoid which is divisible by 2 distinctively and $(B, d)$ is a dq-metric space satisfying the completeness property with the continuous binary operator + . Let $\mathrm{L} \subset A$ be a non-empty set such that $r \neq 0, r+s \neq 0$, for all $r, s \in \mathrm{L}$. Further assume that $\zeta_{1}, \zeta_{2}: L \times L \longrightarrow \mathbb{R}^{+} \cup\{0\}$ are mappings. We determine the stability result of equation (1.1) with these assumptions in the ensuing result.

Theorem 2.1. Suppose $\rho_{1}, \rho_{2}, \theta \in(0, \infty)$ exist with the conditions $\rho_{1} \theta<1$ and $\rho_{2} \theta<1$ and

$$
\zeta_{k}\left(\frac{r}{2}, \frac{s}{2}\right) \leqslant \rho_{k} \zeta_{k}(r, s), \quad r, s \in L, \quad k=1,2, \quad d\left(\frac{1}{2} h(r), \frac{1}{2} h(s)\right) \leqslant \theta d(h(r), h(s)), \quad r, s \in L .
$$

If a mapping $\mathrm{h}: \mathrm{L} \longrightarrow \mathrm{B}$ satisifes the following conditions:

$$
d\left(\frac{h(r) h(s)}{h(r)+h(s)}, h(r+s)\right) \leqslant \zeta_{1}(r, s), \quad d\left(h(r+s), \frac{h(r) h(s)}{h(r)+h(s)}\right) \leqslant \zeta_{2}(r, s)
$$

for all $\mathrm{r}, \mathrm{s} \in \mathrm{L}, \mathrm{r}+\mathrm{s} \in \mathrm{L}$, then a mapping $\mathrm{H}: \mathrm{L} \longrightarrow \mathrm{B}$ exists which is unique, close to $\mathrm{h}$ and is a solution of (1.1) satisfying the ensuing approximations

$$
d(H(r), h(r)) \leqslant \frac{\rho_{1} \zeta_{1}(r, r)}{1-\rho_{1} \theta} \text { and } d(h(r), H(r)) \leqslant \frac{\rho_{2} \zeta_{2}(r, r)}{1-\rho_{2} \theta}
$$

for all $\mathrm{r} \in \mathrm{L}$.

Proof. Replacing $(r, s)$ by $\left(\frac{r}{2}, \frac{r}{2}\right)$ in the assumption, one can find

$$
\mathrm{d}\left(\frac{1}{2} \mathrm{~h}\left(\frac{\mathrm{r}}{2}\right), \mathrm{h}(\mathrm{r})\right) \leqslant \zeta_{1}\left(\frac{\mathrm{r}}{2}, \frac{\mathrm{r}}{2}\right)
$$

for all $r \in L$, and analogously, one can also obtain

$$
d\left(h(r), \frac{1}{2} h\left(\frac{r}{2}\right)\right) \leqslant \zeta_{2}\left(\frac{r}{2}, \frac{r}{2}\right)
$$

for all $r \in$ L. Now, assuming $\Phi(r)=\frac{r}{2}, \mu=\theta, f_{k}(r)=\zeta_{k}\left(\frac{r}{2}, \frac{r}{2}\right), k=1,2$, and $g(r)=\frac{r}{2}$ in Theorem 1.3, then

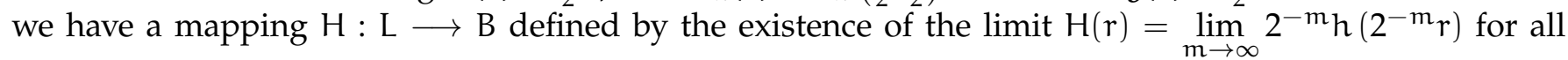
$r \in L$. The mapping $H$ is unique and it satisfies the equation $2^{-1} H\left(2^{-1} r\right)=H(r), r \in L$, such that for all $r \in \mathrm{L}$, we have

$$
d\left((H(r), h(r)) \leqslant \sum_{k=0}^{\infty} \theta^{k} \zeta_{1}\left(\frac{r}{2^{k+1}}, \frac{r}{2^{k+1}}\right) \leqslant \sum_{k=0}^{\infty} \theta^{k} \rho^{k} \zeta_{1}\left(\frac{r}{2}, \frac{r}{2}\right) \leqslant \rho_{1} \zeta_{1}(r, r) \sum_{k=0}^{\infty}\left(\rho_{1} \theta\right)^{k}=\frac{\rho_{1} \zeta_{1}(r, r)}{1-\rho_{1} \theta}\right.
$$


Similarly, one can come up with for all $r \in \mathrm{L}$,

$$
d(h(r), H(r)) \leqslant \frac{\rho_{2} \zeta_{2}(r, r)}{1-\rho_{2} \theta}
$$

Now, for all positive integers $m$, we find that

$$
\begin{aligned}
d\left(\frac{2^{-m} h\left(2^{-m} r\right) 2^{-m} h\left(2^{-m} s\right)}{2^{-m} h\left(2^{-m} r\right)+2^{-m} h\left(2^{-m_{s}}\right)}, 2^{-m} h\left(2^{-m}(r+s)\right)\right) & \leqslant \theta^{m} d\left(\frac{h\left(2^{-m} r\right) h\left(2^{-m_{1}} s\right)}{h\left(2^{-m} r\right)+h\left(2^{-m_{s}} s\right)}, h\left(2^{-m}(r+s)\right)\right) \\
& \leqslant \theta^{m} \zeta_{1}\left(2^{-m} r, 2^{-m} s\right) \\
& \leqslant\left(\rho_{1} \theta\right)^{m} \zeta_{1}(r, s)
\end{aligned}
$$

for all $r, s \in$ L. Allowing $m$ to $\infty$ in the above inequality and since $\rho \theta<1$, we can observe that $H$ satisfies equation (1.1). By similar approach of the arguments as in Theorem 1.3, it is easy to show that this mapping $\mathrm{H}$ is unique, which completes the proof.

In the following corollaries, we apply Theorem 2.1 by considering $L$ to be the set of real numbers containing elements $r, s \in \mathbb{R}$ with the conditions $r \neq 0$ and $r+s \neq 0$.

Corollary 2.2. Suppose that $\mathrm{k} \in(0, \infty)$ be a fixed constant and there exists a constant $\theta \in(0,1)$ such that

$$
d\left(\frac{1}{2} h(r), \frac{1}{2} h(s)\right) \leqslant \theta d(h(r), h(s)), \quad r, s \in L .
$$

Let $\mathrm{h}: \mathrm{L} \longrightarrow \mathrm{B}$ be a mapping satisfing the following inequalities

$$
d\left(\frac{h(r) h(s)}{h(r)+h(s)}, h(r+s)\right) \leqslant k \text { and } d\left(h(r+s), \frac{h(r) h(s)}{h(r)+h(s)}\right) \leqslant k
$$

for all $\mathrm{r}, \mathrm{s} \in \mathrm{L}$. Then the equation (1.1) has a unique solution $\mathrm{H}: \mathrm{L} \longrightarrow \mathrm{B}$ and this solution $\mathrm{H}$ satisfies the following approximations

$$
\mathrm{d}(\mathrm{H}(\mathrm{r}), \mathrm{h}(\mathrm{r})) \leqslant \frac{\mathrm{k}}{1-\theta} \text { and } \mathrm{d}(\mathrm{h}(\mathrm{r}), \mathrm{H}(\mathrm{r})) \leqslant \frac{\mathrm{k}}{1-\theta}
$$

for all $\mathrm{r} \in \mathrm{L}$.

Corollary 2.3. Let $\mathrm{k}_{1} \in(0, \infty)$ be a fixed constant. Let there exist $\theta, \mathrm{a} \in(0, \infty)$ with the condition that $\frac{\theta}{2^{a}}<1$ and

$$
\mathrm{d}\left(\frac{1}{2} \mathrm{~h}(\mathrm{r}), \frac{1}{2} \mathrm{~h}(\mathrm{~s})\right) \leqslant \theta \mathrm{d}(\mathrm{h}(\mathrm{r}), \mathrm{h}(\mathrm{s})), \quad \mathrm{r}, \mathrm{s} \in \mathrm{L} .
$$

Let $\mathrm{h}: \mathrm{L} \longrightarrow \mathrm{B}$ be a mapping satisfies the following inequalities

$$
d\left(\frac{h(r) h(s)}{h(r)+h(s)}, h(r+s)\right) \leqslant k_{1}\left(|r|^{a}+|s|^{a}\right) \quad \text { and } d\left(h(r+s), \frac{h(r) h(s)}{h(r)+h(s)}\right) \leqslant k_{1}\left(|r|^{a}+|s|^{a}\right)
$$

for all $\mathrm{r}, \mathrm{s} \in \mathrm{L}$. Then the equation (1.1) has a unique solution $\mathrm{H}: \mathrm{L} \longrightarrow \mathrm{B}$ and this solution $\mathrm{H}$ satisfies the following approximations

for all $r \in \mathrm{L}$.

$$
\mathrm{d}(\mathrm{H}(\mathrm{r}), \mathrm{h}(\mathrm{r})) \leqslant \frac{2 \mathrm{k}_{1}}{2^{\mathrm{a}}-\theta}|\mathrm{r}|^{\mathrm{a}} \text { and } \mathrm{d}(\mathrm{h}(\mathrm{r}), \mathrm{H}(\mathrm{r})) \leqslant \frac{2 \mathrm{k}_{1}}{2^{\mathrm{a}}-\theta}|\mathrm{r}|^{\mathrm{a}}
$$

Corollary 2.4. Let $\mathrm{k}_{\in}(0, \infty) 0$ be a fixed real constant. Let there exist $\theta, \mathrm{a}, \mathrm{b} \in(0, \infty)$ with the condition that $\mathrm{c}=\mathrm{a}+\mathrm{b}$ and $\frac{\theta}{2^{\mathrm{c}}}<1$ and

$$
d\left(\frac{1}{2} h(r), \frac{1}{2} h(s)\right) \leqslant \theta d(h(r), h(s)), \quad r, s \in L
$$


Let $\mathrm{h}: \mathrm{L} \longrightarrow \mathrm{B}$ be a mapping satisfies the following inequalities

$$
d\left(\frac{h(r) h(s)}{h(r)+h(s)}, h(r+s)\right) \leqslant k_{1}\left(|r|^{a}|s|^{b}\right) \quad \text { and } d\left(h(r+s), \frac{h(r) h(s)}{h(r)+h(s)}\right) \leqslant k_{1}\left(|r|^{a}|s|^{b}\right)
$$

for all $\mathrm{r}, \mathrm{s} \in \mathrm{L}$. Then the equation (1.1) has a unique solution $\mathrm{H}: \mathrm{L} \longrightarrow \mathrm{B}$ and this solution $\mathrm{H}$ satisfies the following approximations

$$
\mathrm{d}(\mathrm{H}(\mathrm{r}), \mathrm{h}(\mathrm{r})) \leqslant \frac{\mathrm{k}_{2}}{2^{\mathrm{c}}-\theta}|\mathrm{r}|^{\mathrm{c}} \text { and } \mathrm{d}(\mathrm{h}(\mathrm{r}), \mathrm{H}(\mathrm{r})) \leqslant \frac{\mathrm{k}_{2}}{2^{\mathrm{c}}-\theta}|\mathrm{r}|^{\mathrm{c}}
$$

for all $\mathrm{r} \in \mathrm{L}$.

\section{Discussion of the stability results of equation (1.1) obtained}

In Theorem 1.3, the stability results of fundamental Cauchy additive equation are obtained by considering a common control mapping only as an upper bound. But in this study, we have extended the generalized stability by considering different upper bounds in the corollaries by taking $L$ to be $\mathbb{R}$ such that $r \neq 0$ and $r+s \neq 0$ to avoid singularities to achieve the main results.

\section{Conclusion}

So for in the literature, several functional equations in rational form are dealt to investigate their stabilities in the framework of different spaces. This is our first attempt to consider dq-metric space to analyze the validity of stableness of equation (1.1). The dq-metric spaces are useful in logic programming, theoretical computer science and electronics and hence the stability results obtained in this study can be utilized to approach problems arising in the above fields $[1,2,19]$.

\section{Acknowledgment}

The first and second authors are supported by The Research Council, Oman (Under Project proposal ID: BFP/RGP/CBS/18/099).

\section{References}

[1] C. T. Aage, J. N. Salunke, The results on fixed points in dislocated and dislocated quasi-metric space, Appl. Math. Sci., 2 (2008), 2941-2948. 4

[2] C. T. Aage, J. N. Salunke, Some results of fixed point theorem in dislocated quasi-metric spaces, Bull. Marathwada Math. Soc., 9 (2008), 1-5. 4

[3] M. R. Abdollahpour, R. Aghayari, M. T. Rassias, Hyers-Ulam stability of associated Laguerre differential equations in a subclass of analytic functions, J. Math. Anal. Appl., 437 (2016), 605-612. 1

[4] M. R. Abdollahpour, M. T. Rassias, Hyers-Ulam stability of hypergeometric differential equations, Aequa. Math., 93 (2019), 691-698. 1

[5] J. Aczél, J. Dhombres, Functional Equations in Several Variables, Cambridge University Press, 31, (1989). 1

[6] A. Amini-Harandi, Metric-like spaces, partial metric spaces and fixed points, Fixed Point Theory Appl., 2012 (2012), 10 pages. 1

[7] T. Aoki, On the stability of the linear transformation in Banach spaces, J. Math. Soc. Japan, 2 (1950), 64-66. 1

[8] A. Bodaghi, B. V. Senthil Kumar, Estimation of inexact reciprocal-quintic and reciprocal-sextic functional equations, Mathematica, 59 (2017), 3-14. 1

[9] D. G. Bourgin, Classes of transformations and bordering transformations, Bull. Amer. Math. Soc., 57 (1951), 223-237. 1

[10] J. Brzdęk, On a method of proving the Hyers-Ulam stability of functional equations on restricted domains, Aust. J. Math. Anal. Appl., 6 (2009), 1-10. 1

[11] P. W. Cholewa, Remarks on the stability of functional equations, Aequationes Math., 27 (1984), 76-86. 1

[12] K. Ciepliński, On Ulam stability of a functional equation, Results Math., 75 (2020), 1-11. 1

[13] S. Czerwik, Functional Equations and Inequalities in Several Variables, World Scientific, (2002). 1

[14] A. Ebadian, M. E. Gordji, H. Khodaei, R. Saadati, G. Sadeghi, On the stability of an m-variables functional equation in random normed spaces via fixed point method, Discrete Dyn. Nat. Soc., 2012 (2012), 13 pages. 1 
[15] Z. Gajda, On the stability of additive mappings, Int. J. Math. Math. Sci., 14 (1991), 431-434. 1

[16] P. Găvruta, A generalization of the Hyers-Ulam-Rassias stability of approximately additive mapppings, J. Math. Anal. Appl., 184 (1994), 431-436. 1

[17] B. Hejmej, Stability of functional equations in dislocated quasi-metric spaces, Ann. Math. Sil., 32 (2018), 215-225. 1

[18] H. Dutta, B. V. Senthil Kumar, Classical stabilities of an inverse fourth power functional equation, J. Interdisciplinary Math., 22 (2019), 1061-1070. 1

[19] P. Hitzler, Generalized metrics and topology in logic programming semantics, Ph.D. Thesis, School of Mathematics, Applied Mathematics and Statistics, National University Ireland, University College Cork, (2001). 4

[20] D. H. Hyers, On the stability of the linear functional equation, Proc. Nat. Acad. Sci. U.S.A., 27 (1941), 222-224. 1

[21] D. H. Hyers, G. Isac, T. M. Rassias, Stability of Functional Equations in Several Variables, Birkhäuser, Boston, (1998). 1

[22] D. H. Hyers, T. M. Rassias, Approximate homomorphisms, Aequationes Math., 44 (1992), 125-153. 1

[23] M. Janfada, G. Sadeghi, Generalized Hyers-Ulam stability of a quadartic functional equation with involution in quasi- $\beta$ normed spaces, J. Appl. Math. Informatics, 29 (2011), 1421-1433. 1

[24] S.-M. Jung, Hyers-Ulam-Rassias stability of Functional Equations in Nonlinear Analysis, 48, Springer, New York, (2011). 1

[25] S.-M. Jung, D. Popa, M. T. Rassias, On the stability of the linear functional equation in a single variable on complete metric groups, J. Global Optim., 59 (2014), 165-171. 1

[26] S.-M. Jung, M. T. Rassias, A linear functional equation of third order associated to the Fibonacci numbers, Abstr. Appl. Anal., 2014 (2014), 7 pages.

[27] S.-M. Jung, M. T. Rassias, C. Mortici, On a functional equation of trigonometric type, Appl. Math. Comput., 252 (2015), 294-303. 1

[28] P. Kannappan, Functional Equations and Inequalities with Applications, Springer, New York, (2009). 1

[29] G. H. Kim, Y.-H. Lee, , Demonstr. Math., 53 (2020), 1-7. 1

[30] S. O. Kim, B. V. Senthil Kumar, A. Bodaghi, Approximation on the reciprocal-cubic and reciprocal-quartic functional equations in non-Archimedean fields, Adv. Difference Equ., 2017 (2017), 12 pages. 1

[31] Y.-H. Lee, On the Hyers-Ulam-Rassias stability of a general quintic functional equation and a general sextic functional equation, Mathematics, 7 (2019), 1-14. 1

[32] Y.-H. Lee, S.-M. Jung, M. T. Rassias, On an n-dimensional mixed type additive and quadratic functional equation, Appl. Math. Comput., 228 (2014), 13-16. 1

[33] Y.-H. Lee, S.-M. Jung, M. T. Rassias, Uniqueness theorems on functional inequalities concerning cubic-quadratic-additive equation, J. Math. Inequal., 12 (2018), 43-61.

[34] C. Mortici, M. T. Rassias, S.-M. Jung, On the stability of a functional equation associated with the Fibonacci numbers, Abstr. Appl. Anal., 2014 (2014), 6 pages. 1

[35] N. B. Okelo, Properties of complete metric spaces and their applications to computer security, information and communication technology, Evolving Trends Eng. Technol., 1 (2014), 23-28. 1

[36] C. Park, M. T. Rassias, Additive functional equations and partial multipliers in $\mathrm{C}^{*}$-algebras, Rev. R. Acad. Cienc. Exactas Fís. Nat. Ser. A Mat. RACSAM, 113 (2019), 2261-2275. 1

[37] M. U. Rahman, M. Sarwar, Some new fixed point theorems in dislocated quasi-metric spaces, Palest. J. Math., 5 (2016), 171-176. 1

[38] J. M. Rassias, On approximately of approximately linear mappings by linear mappings, J. Funct. Anal., 46 (1982), $126-130$. 1

[39] J. M. Rassias, E. Thandapani, K. Ravi, B. V. Senthil Kumar, Functional Equations and Inequalities: Solutions and Stability Results, Word Scientific Publishing Company, Singapore, (2017). 1

[40] T. M. Rassias, On the stability of the linear mapping in Banach spaces, Proc. Amer. Math. Soc., 72 (1978), 297-300. 1

[41] T. M. Rassias, Functional Equations and Inequalities, Kluwer Academic Publishers, (2000). 1

[42] K. Ravi, J. M. Rassias, B. V. Senthil Kumar, Ulam stability of generalized reciprocal functional equation in several variables, Int. J. Appl. Math. Stat., 19 (2010), 1-19. 1

[43] K. Ravi, B. V. Senthil Kumar, Ulam-Gavruta-Rassias stability of Rassias reciprocal functional equation, Global J. Appl. Math. Sci., 3 (2010), 57-79. 1

[44] K. Ravi, B. V. Senthil Kumar, Stability and geometrical interpretation of reciprocal type functional equation, Asian J. Curr. Eng. Maths, 1 (2012), 300-304. 1

[45] R. Saadati, G. Sadeghi, T. M. Rassias, Approximate generalized additive mappings in proper multi-CQ*-algebras, Filomat, 28 (2014), 677-694. 1

[46] G. Sadeghi, A fixed point approach to stability of functional equations in modular spaces, Bull. Malays. Math. Sci. Soc., 37 (2014), 333-344.

[47] G. Sadeghi, M. Nazarianpoor, J. M. Rassias, Solution and stability of quattuorvigintic functional equation in intuitionistic fuzzy normed spaces, Iran. J. Fuzzy Syst., 15 (2018), 13-30. 1

[48] P. K. Sahoo, P. Kannappan, Introduction to Functional Equations, CRC Press, (2011). 1

[49] M. Sarwar, M. U. Rahman, G. Ali, Some fixed point results in dislocated quasi-metric (dq-metric) spaces, J. Inequal. Appl.J. Inequal. Appl., 2014 (2014), 11 pages. 1

[50] B. V. Senthil Kumar, H. Dutta, Fuzzy stability of a rational functional equation and its relevance to system design, Int. J. 
Gen. Syst., 48 (2019), 157-169. 1

[51] B. V. Senthil Kumar, H. Dutta, Approximation of multiplicative inverse undecic and duodecic functional equations, Math. Methods Appl. Sci., 42 (2019), 1073-1081. 1

[52] B. V. Senthil Kumar, H. Dutta, S. Sabarinathan, Approximation of a system of rational functional equations of three variables, Int. J. Appl. Comput. Math., 5 (2019), 1-16. 1

[53] B. V. Senthil Kumar, H. Dutta, Khalifa Al-Shaqsi, On a functional equation arising from subcontrary mean and its pertinences, Advances in Intelligent Systems and Computing, 4th International Conference on Computational Mathematics and Engineering Sciences, (2020), 241-247. 1

[54] B. V. Senthil Kumar, K. Al-Shaqsi, H. Dutta, Classical stabilities of multiplicative invese difference and adjoint functional equations, Adv. Difference Equ., 2020 (2020), 9 pages. 1

[55] B. V. Senthil Kumar, H. Dutta, S. Sabarinathan, Fuzzy approximations of a multiplicative inverse cubic functional equation, Soft Comput., 24 (2020), 13285-13292. 1

[56] S. M. Ulam, Problems in Modern Mathematics, Science Editions John Wiley \& Sons, New York, (1964). 1

[57] J. Wang, Some further generalization of the Hyers-Ulam-Rassias stability of functional equations, J. Math. Anal. Appl., 263 (2001), 406-423. 1 